\title{
Tendinite do manguito rotador em operadores de caixa de supermercado: contributos para a vigilância de saúde
}

\author{
Rotator cuff tendinitis in supermarket cashiers: \\ contributions to health surveillance
}

\author{
Rita Silva-Pereira', Florentino Serranheira², Fátima Lopes³ ${ }^{3}$ Ricardo Ribeiro ${ }^{4}$, António Sousa-Uva²
}

\begin{abstract}
RESUMO | Contexto: A tendinite do manguito rotador (TMR) é frequente em operadores de caixa de supermercado devido às exigências do trabalho. É, de facto, frequente a realização de movimentos repetitivos de abdução e flexão dos membros superiores, muitas vezes em carga, que podem causar inflamação dos tecidos periarticulares do ombro, desencadeando sinais e sintomas de TMR. Objetivos: Conhecer a prevalência de TMR em operadores de caixa de supermercado por meio da avaliação de sintomas, sinais e ecografia. Métodos: Participaram 44 trabalhadores com idades entre 28 e 47 anos. Eles preencheram um questionário de queixas autorreferidas aos ombros e realizaram exame objetivo (manobras do arco doloroso, do conflito subacromial e abdução resistida do ombro) e ecografia de tecidos moles. Resultados: Identificaram-se queixas de dor no ombro em 26 operadores à direita (59,1\%) e em $16(36,4 \%)$ à esquerda. Ao exame objetivo, 15 e 11 trabalhadores (34,1\% à direita e $25,0 \%$ à esquerda, respetivamente) apresentaram positividade nas três manobras. Na ultrassonografia foram encontradas alterações no ombro direito e esquerdo em $16 \mathrm{e}$ 17 operadores (36,4 e 38,6\%, respetivamente). Não foi encontrada concordância estatística entre as três abordagens. A "manobra de abdução resistida do ombro" revelou boa relação com as queixas de dor no ombro $\left(\chi^{2}{ }_{\text {Wald }}(1)=7,260 ; p=0,007\right)$. Obtiveram-se resultados semelhantes de associação entre essa manobra e a avaliação ecográfica $\left(\chi_{\text {Wald }}^{2}(1)=6,854 ; \mathrm{p}=0,009\right.$; ROC $=0,714$, sensibilidade 75\%; especificidade 67,9\%). Conclusões: Os resultados obtidos sugerem que a "manobra de abdução resistida do ombro" é preditiva de TMR na vigilância médica desses trabalhadores. Tal deverá ser verificado em futuros estudos, designadamente envolvendo amostras de maiores dimensões.
\end{abstract}

Palavras-chave I medicina do trabalho; tendinopatia; LER-DORT; vigilância da saúde.

ABSTRACT | Background: Rotator cuff tendinitis (RCT) is common among supermarket cashiers due to their work demands. Repetitive movements of shoulder abduction and flexion are frequent, which might cause inflammation of the periarticular tissues of the shoulder, triggering signs and symptoms of RCT. Objectives: To investigate the prevalence of RCT in supermarket cashiers by means of assessment of symptoms, signs and ultrasound. Methods: Forty-four workers aged 28 to 47 years old participated in the study. The participants were subjected to a self-report shoulder complaints questionnaire, clinical examinations ("painful arc test", "empty can test" and "Hawkins-Kennedy impingement test") and soft tissue ultrasound. Results: A total of 26 participants reported shoulder pain on the right side (59.1\%) and 16 (36.4\%) on the left side. During clinical assessment, 15 and 11 workers (34.1\% right and $25.0 \%$ left, respectively) had positive results on all three tests. Ultrasound showed alterations in the right and left shoulder in 16 and 17 participants ( 36.4 and $38.6 \%$, respectively). No statistical correlation was found between the three approaches. The "empty can test" exhibited strong correlation with shoulder pain $\left(\chi_{\text {wald }}^{2}(1)=7.260, p=0.007\right)$. Similar results were obtained for the association between this test and ultrasound $\left(\chi^{2}{ }_{\text {wald }}(1)=6.854, \mathrm{p}=0.009, \mathrm{ROC}=0.714\right.$, sensitivity $75 \%$, specificity $\left.67.9 \%\right)$. Conclusions: The results suggest that the "empty can test" is predictive of RCT in the medical surveillance of supermarket cashiers. This finding should be verified in future studies, especially with larger samples.

Keywords I occupational medicine; tendinopathy; musculoskeletal pain; public health surveillance.

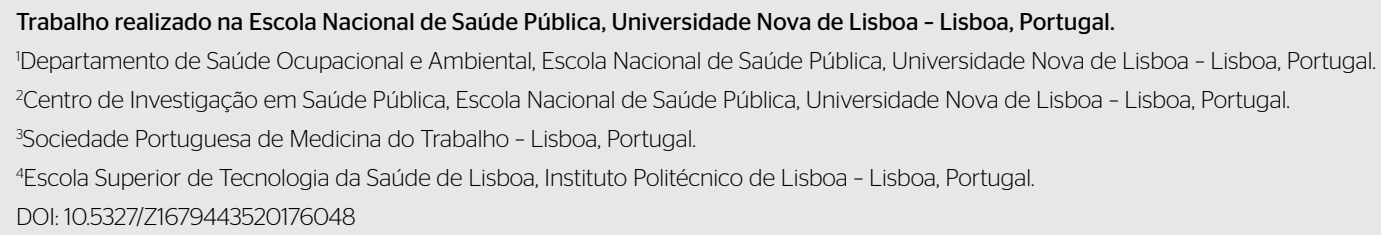




\section{INTRODUÇÃO}

Os distúrbios osteomusculares relacionados ao trabalho (DORT) encontram-se entre as patologias mais predominantes na população em geral e atingem igualmente uma elevada prevalência em contexto laboral. Nos países industrializados, essas patologias dos membros superiores constituem as doenças profissionais mais frequentes, contribuindo para o número crescente de trabalhadores com capacidade de trabalho reduzida. $\mathrm{O}$ manguito rotador é constituído por quatro músculos (supraespinoso, infraespinoso, subescapular e redondo menor), e respetivos tendões, que se encontram posicionados em torno da articulação do ombro, conferindolhe estabilidade e possibilitando a realização de movimentos de elevação e rotação do membro superior. A tendinite do manguito rotador (TMR) constitui a terceira causa de lesão musculoesquelética, sendo apenas ultrapassada pela lombalgia e pela cervicalgia ${ }^{1}$. Afeta mais frequentemente o tendão do músculo supraespinoso, na vizinhança da sua inserção com a tuberosidade maior do úmero, imediatamente abaixo do bordo externo do acrómio. A incidência das lesões aumenta com a idade, sendo, no entanto, os microtraumatismos e a sobrecarga profissional e/ou desportiva importantes causas em qualquer faixa etária ${ }^{1-3}$.

A etiologia da TMRé multifatorial, resultando da combinação de fatores intrínsecos, extrínsecos e ambientais ${ }^{4}$. O desenvolvimento de dor no ombro depende tanto da intensidade quanto da frequência e da duração das atividades laborais (exemplos: posturas articulares extremas, manipulação de cargas e movimentos repetitivos do membro superior), assim como de alguns fatores de risco psicossociais $^{5-7}$. Os operadores de caixa de supermercado apresentam prevalência elevada de DORT dos membros superiores, particularmente nos ombros e nas mãos, sendo os movimentos repetitivos e as posturas articulares extremas considerados os seus principais fatores de risco ${ }^{8}$. Um estudo realizado por Moosmayer revelou que 7,6\% dos indivíduos apresentavam rotura do manguito rotador (RMR) assintomática, existindo associação significativa com a idade ${ }^{9}$. Outros estudos confirmam a importância dos fatores de risco psicossociais, quer os individuais (exemplo: existência de antecedentes patológicos de depressão), quer os organizacionais relacionados com o conteúdo e o contexto de trabalho (exemplo: baixo nível de controlo sobre o trabalho), assim como os fatores de risco relacionados com a atividade (exemplos: movimentos repetitivos, posturas extremas e aplicação de força), na patologia dolorosa do ombro ${ }^{10-12}$.

Os DORT do ombro são com frequência assintomáticos. Um estudo realizado em $2012^{1}$ revelou que, nos doentes com diagnóstico de RMR unilateral, a prevalência de casos assintomáticos no membro superior contralateral era de 35,5\%. Um número considerável de doentes assintomáticos desenvolveu sintomatologia passado um curto período de tempo de follow-up e apresentou deterioração da função da articulação do ombro.

A principal característica clínica sugestiva de patologia periarticular do ombro é o ritmo inflamatório da dor. A prevalência das queixas autorreferidas ao ombro está estimada entre 16 e $26 \%^{2}$. A TMR, a tendinite da longa porção do bíceps braquial (TLPBB) e a bursite subacromial constituem as principais causas de dor referida ao ombro ${ }^{13,14}$, a qual está associada à diminuição do estado de saúde e da qualidade de vida, sendo desejável o desenvolvimento de medidas para a sua prevenção primária ou secundária ${ }^{15}$.

O diagnóstico da TMRé clínico, não existindo, contudo, ao exame objetivo, uma manobra gold standard que permita o diagnóstico isolado desse tipo de patologia ${ }^{16}$. Um estudo realizado em $19922^{17}$ revela sensibilidade de $91 \%$ e especificidade de $75 \%$ para as manobras diagnósticas baseadas em sinais clínicos, designadamente, dor desencadeada pela abdução resistida do ombro. Em caso de dúvida, recorrese aos exames de imagem, nomeadamente à ecografia dos tecidos moles ${ }^{18,19}$. A utilização de outras técnicas de imagem como a tomografia axial computorizada (TAC) e a ressonância magnética nuclear (RMN) tem influenciado a forma como a ecografia é utilizada na avaliação do aparelho musculoesquelético ${ }^{20}$. A RMN é, atualmente, o método imagiológico de referência para o estudo das lesões do ombro. $\mathrm{O}$ uso combinado das duas técnicas apresenta vantagens diagnósticas adicionais ${ }^{13}$. A ultrassonografia (US), além da elevada sensibilidade na identificação de calcificações e de possibilitar guiar procedimentos diagnósticos e/ou terapêuticos invasivos, pode ser utilizada na presença de material pós-operatório metálico e em casos de claustrofobia ${ }^{21}$. Além disso, o baixo custo, a acessibilidade e a inocuidade conferem-lhe vantagens em relação às restantes técnicas de imagem ${ }^{2,22}$ que exigem, todavia, experiência do examinador ${ }^{1,13,22-25}$. Entre as alterações estruturais mais comuns destacam-se: 
1. calcificações nos tendões do manguito rotador do ombro, com prevalência de 2,7 a $7,5 \%$ na população geral, podendo ocorrer de forma sintomática ou assintomática ${ }^{26,27}$;

2. tendinose do manguito rotador;

3. roturas parciais ou totais do supraespinoso, infraespinoso e subescapular ${ }^{1,13,28,29}$; e

4. TLPBB, muitas vezes associada à tendinite do supraespinoso e do subescapular, o que justifica, na presença dessas patologias, avaliar a longa porção do bíceps braquial, estrutura que proporciona estabilidade adicional à articulação do ombro ${ }^{30}$.

A inexistência de programas de vigilância médica bem estruturados em medicina do trabalho que permitam a identificação de DORT dos membros superiores pode ser a causa da subestimação do risco e conduzir à falta de informação necessária para a adoção de medidas preventivas adequadas ${ }^{31}$.

O presente trabalho objetivou o estudo da prevalência de TMR em operadores de caixa de supermercado utilizando três metodologias: um questionário de queixas autorreferidas, o exame objetivo e a ultrassonografia com vista a determinar adequados protocolos de vigilância médica desses trabalhadores.

\section{MÉTODOS}

O presente estudo, transversal, abrange uma amostra de conveniência de 50 operadores de caixa de um grande hipermercado da região de Lisboa que é representativa (margem de erro de 10\% e nível de significância de 90\%) do número de trabalhadores da empresa em estudo $(\mathrm{n}=158)$. Os seguintes critérios de inclusão e de exclusão determinaram a eliminação da amostra de seis indivíduos:

1. critérios de inclusão: operadores de caixa de um hipermercado da região da Grande Lisboa que aceitaram participar voluntariamente no estudo e responderam a uma adaptação portuguesa do questionário nórdico musculoesquelético (QNM) que inclui as principais dimensões do questionário original, nomeadamente a avaliação da presença/ausência de sintomas musculoesqueléticos nos diversos segmentos anatómicos nos últimos 7 dias e nos últimos 12 meses, uma dimensão sobre o estado de saúde e uma nova dimensão sobre a relação entre a sintomatologia musculoesquelética e os principais fatores de risco da situação de trabalho do respondente ${ }^{32,33}$.

2. critérios de exclusão: antecedentes patológicos de fraturas do ombro/úmero; antecedentes patológicos de traumatismos, entorse ou luxação do ombro; e antecedentes de fibromialgia.

Todos os participantes assinaram um termo de consentimento informado. Para a obtenção das características sociodemográficas e queixas autorreferidas foi aplicado um questionário de autopreenchimento. Os participantes foram, depois, submetidos a exame objetivo e estudo ultrassonográfico em ambos os ombros, tendo sido assegurados os procedimentos que garantiram que os investigadores não tivessem conhecimento prévio de qualquer dos resultados em qualquer momento do estudo.

Para a obtenção das características sociodemográficas, das características da atividade laboral, da situação de saúde e das queixas autorreferidas ligadas ao trabalho (dor no ombro), foi aplicado o $\mathrm{QNM}^{6,32,33}$. Consideraram-se "casos sintomáticos" aqueles em que existiam queixas autorreferidas de dor no ombro e "casos assintomáticos" aqueles que apresentavam alterações à análise ecográfica, mas sem queixas autorreferidas.

A sintomatologia foi caracterizada de acordo com a intensidade e a frequência em escalas de 0 a 10 e 1 a 3, respetivamente. Os participantes passaram, em seguida, à segunda fase do estudo, que consistiu nas seguintes observações clínicas: 1. avaliação dos dois ombros, incluindo a amplitude dos movimentos e a existência (ou não) de dor à mobilização por meio da realização dos seguintes testes diagnósticos padronizados: manobra do arco doloroso, manobra de abdução resistida do ombro e manobra de conflito subacromial;

2. avaliação ecográfica de ambos os ombros, tendo em vista a caracterização das estruturas distais do manguito rotador (tendão subescapular, supraespinoso e infraespinoso) e da longa porção do bíceps braquial, em termos de ecogenicidade, ecotextura e espessura.

A realização da manobra do arco doloroso, no contexto da medicina do trabalho, deve iniciar-se por pedir ao trabalhador que eleve os braços em abdução até $180^{\circ}$. Na presença de TMR, o trabalhador referirá dor à mobilização ativa ao longo de um arco entre 60 e $120^{\circ}$, e apresentará limitação da abdução 
do membro superior. Na mobilização passiva, a abdução será tipicamente completa e muito menos dolorosa do que na mobilização ativa. Na manobra da abdução resistida do ombro, o membro superior é colocado passivamente a $90^{\circ}$ de abdução com o polegar apontando para baixo, após o que o médico aplica força sobre o membro superior para baixo enquanto o trabalhador deve resistir contrariando o movimento. $\mathrm{Na}$ presença de inflamação do manguito rotador, a manobra despertará dor intensa no ombro afetado. Nos casos em que a inflamação é intensa ou existe rotura do tendão, o trabalhador poderá ser incapaz de manter o braço em abdução, mesmo na ausência de resistência por parte do médico. Na manobra de conflito subacromial, útil para o diagnóstico quer da TMR quer da bursite subacromial, solicita-se ao trabalhador que coloque a palma da mão sobre o ombro contralateral, com o cotovelo à altura dos ombros, e mantenha essa posição enquanto o médico aplica força sobre o cotovelo para baixo. Na manobra positiva, o trabalhador referirá dor no ombro em observação ${ }^{19,20}$.

A aquisição das imagens ecográficas foi realizada de acordo com as guidelines técnicas de ultrassonografia musculoesquelética recomendadas pela European Society of Musculoskeletal Radiology:

- longa porção do bíceps: braço ao longo do corpo em ligeira rotação interna, cotovelo fletido a $90^{\circ}$ e mão em supinação;

- subescapular: partindo do posicionamento anterior, realizar rotação externa máxima do membro superior, mantendo o cotovelo fixo ao nível superior da crista ilíaca;

- supraespinoso: face dorsal da mão sobre o bolso posterior das calças (rotação interna forçada/manobra de stress), não devendo existir qualquer espaço entre o cotovelo $\mathrm{e}$ a parede torácica lateral;

- infraespinoso: mão apoiada sobre o ombro oposto a estudar.

Os trabalhadores foram instruídos a não reportar sintomas durante a realização do exame objetivo e da ultrassonografia.

Para a análise estatística utilizou-se o SPSS (Statistical Package for the Social Sciences) versão 20.0 para Windows. Usaram-se medidas de estatística descritiva (frequências absolutas e relativas, médias e respetivos desvios-padrão) e estatística inferencial. O nível de significância para aceitar ou rejeitar a hipótese nula foi fixado em $(\alpha) \leq 0,05$. Usou-se o teste binomial quando se compararam duas proporções independentes, o teste de McNemar para duas proporções emparelhadas, o teste do $\chi^{2}$ de independência e a regressão logística. O pressuposto do $\chi^{2}$ de que não deve haver mais de $20,0 \%$ das células com frequências esperadas inferiores a 5 foi analisado. Nas situações em que esse pressuposto não estava satisfeito, usou-se o teste do $\chi^{2}$ por simulação de Monte Carlo. As diferenças foram analisadas com o apoio dos resíduos ajustados normalizados. Para as variáveis com dois níveis $(2 \times 2)$ usou-se o teste de Fisher.

\section{RESULTADOS}

A amostra foi constituída por 44 operadores de caixa, dos quais $89,0 \%(n=39)$ eram do género feminino e $11,0 \%$ $(\mathrm{n}=5)$, do género masculino. A média de idades foi de 37 anos, o peso, de $68 \mathrm{~kg}$ e $88 \%$ eram destros. A antiguidade média na profissão era de 14,5 anos.

\section{QUEIXAS AUTORREFERIDAS DE DOR NO OMBRO}

O desconforto ou a dor afetavam mais da metade dos inquiridos, sendo a dor localizada no ombro direito a segunda queixa mais frequente $(59,1 \%)$, logo atrás da cervicalgia $(63,6 \%)$. Relativamente à intensidade da dor, a localizada no ombro foi, em média, mais intensa à esquerda, sendo a frequência semelhante bilateralmente (Tabela 1).

Quando solicitados a identificar as atividades desenvolvidas em relação às queixas autorreferidas, os operadores indicaram a manipulação de cargas com peso superior a $4 \mathrm{~kg}$ $(89,5 \%)$, a repetitividade do movimento dos braços $(84,7 \%)$ e do movimento das mãos/dedos (80,0\%) como muito (ou totalmente) relacionadas com a sintomatologia (Tabela 2).

Tabela 1. Dor no ombro: intensidade e frequência.

\begin{tabular}{|c|c|c|c|c|c|}
\hline & $\mathrm{n}$ & Mínimo & Máximo & Média & $\begin{array}{l}\text { Desvio } \\
\text { padrão }\end{array}$ \\
\hline \multicolumn{6}{|l|}{ Ombro direito } \\
\hline Intensidade & 21 & 1 & 10 & 5,38 & 2,334 \\
\hline Frequência & 21 & 1 & 8 & 2,05 & 1,627 \\
\hline \multicolumn{6}{|c|}{ Ombro esquerdo } \\
\hline Intensidade & 15 & 3 & 10 & 5,93 & 2,187 \\
\hline Frequência & 15 & 1 & 3 & 2,00 & 0,926 \\
\hline
\end{tabular}


Ao exame objetivo, 34,1\% $(\mathrm{n}=15)$ dos participantes apresentaram todas as manobras para TMR positivas à direita, e $25,0 \%(n=11)$, à esquerda.

À ecografia, a prevalência de alterações sugestivas de TMR foi ligeiramente maior no ombro esquerdo $(38,6 \%)$ do que no ombro direito $(36,4 \%)$, não sendo a diferença estatisticamente significativa.

O tendão mais atingido foi o do supraespinoso, tendose registrado três casos de lesões do subescapular e quatro do infraespinoso (Tabela 3).

A relação entre a idade e a TMR foi estatisticamente significativa tanto à direita quanto à esquerda (respetivamente, $\mathrm{p}<0,038$ e $\mathrm{p}<0,010)$. Existia uma proporção significativamente mais elevada de participantes no escalão etário superior a 40 anos com TMR (Tabela 4). Todos os participantes com avaliação ecográfica positiva eram do género feminino.

A avaliação ecográfica permitiu, ainda, a identificação de dois casos de bursite subacromial.

Todos os trabalhadores com TMR, tanto à direita quanto à esquerda, eram destros ou ambidestros, respetivamente $87,5 / 12,5 \%$ e $88,2 / 11,8 \%$.

Quando consideradas "todas as manobras positivas", não se encontrou relação significativa entre as queixas

Tabela 2. Relação das atividades com a sintomatologia.

\begin{tabular}{|c|c|c|c|c|c|}
\hline & & Sem relação & $\begin{array}{l}\text { Pouco } \\
\text { relacionado }\end{array}$ & $\begin{array}{c}\text { Muito } \\
\text { relacionado }\end{array}$ & $\begin{array}{l}\text { Totalmente } \\
\text { relacionado }\end{array}$ \\
\hline \multirow{2}{*}{ Trabalho sentado } & Freq. & 7 & 9 & 12 & 7 \\
\hline & $\%$ & 20,0 & 25,7 & 34,3 & 20,0 \\
\hline \multirow{2}{*}{ Trabalho de pé } & Freq. & 6 & 11 & 11 & 7 \\
\hline & $\%$ & 17,1 & 31,4 & 31,4 & 20,0 \\
\hline \multirow{2}{*}{ Braços acima da altura dos ombros } & Freq. & 9 & 7 & 7 & 12 \\
\hline & $\%$ & 25,7 & 20,0 & 20,0 & 34,3 \\
\hline \multirow{2}{*}{ Inclinar o tronco } & Freq. & 5 & 12 & 10 & 10 \\
\hline & $\%$ & 13,5 & 32,4 & 27,0 & 27,0 \\
\hline \multirow{2}{*}{ Rodar o tronco } & Freq. & 5 & 12 & 8 & 9 \\
\hline & $\%$ & 14,7 & 35,3 & 23,5 & 26,5 \\
\hline \multirow{2}{*}{ Repetitividade do movimento dos braços } & Freq. & 3 & 3 & 4 & 29 \\
\hline & $\%$ & 7,7 & 7,7 & 10,3 & 74,4 \\
\hline \multirow{2}{*}{ Repetitividade do movimento das mãos } & Freq. & 5 & 3 & 5 & 27 \\
\hline & $\%$ & 12,5 & 7,5 & 12,5 & 67,5 \\
\hline \multirow{2}{*}{ Precisão com os dedos } & Freq. & 6 & 6 & 11 & 17 \\
\hline & $\%$ & 15,0 & 15,0 & 27,5 & 42,5 \\
\hline \multirow{2}{*}{ Aplicar força com as mãos } & Freq. & 6 & 4 & 6 & 23 \\
\hline & $\%$ & 15,4 & 10,3 & 15,4 & 59,0 \\
\hline \multirow{2}{*}{ Manipular cargas 1-4 kg } & Freq. & 4 & 4 & 8 & 22 \\
\hline & $\%$ & 10,5 & 10,5 & 21,1 & 57,9 \\
\hline \multirow{2}{*}{ Manipular cargas $>4$ kg } & Freq. & 2 & 2 & 8 & 26 \\
\hline & $\%$ & 5,3 & 5,3 & 21,1 & 68,4 \\
\hline \multirow{2}{*}{ Levantar e deslocar cargas 10-20 kg } & Freq. & 7 & 3 & 5 & 22 \\
\hline & $\%$ & 18,9 & 8,1 & 13,5 & 59,5 \\
\hline \multirow{2}{*}{ Levantar e deslocar cargas $>10-20$ kg } & Freq. & 9 & 4 & 4 & 18 \\
\hline & $\%$ & 25,7 & 11,4 & 11,4 & 51,4 \\
\hline
\end{tabular}

Freq.: frequência. 
Tabela 3. Resultados do exame objetivo e da avaliação ecográfica.

\begin{tabular}{|c|c|c|c|c|}
\hline \multirow{2}{*}{ TMR } & \multicolumn{2}{|c|}{ Negativo } & \multicolumn{2}{|c|}{ Positivo } \\
\hline & Freq. & $\%$ & Freq. & $\%$ \\
\hline \multicolumn{5}{|c|}{ Todas as manobras positivas } \\
\hline MSD & 29 & 65,9 & 15 & 34,1 \\
\hline MSE & 33 & 75,0 & 11 & 25,0 \\
\hline \multicolumn{5}{|c|}{ Manobra do arco doloroso } \\
\hline MSD & 23 & 52,3 & 21 & 47,7 \\
\hline MSE & 24 & 54,5 & 20 & 45,5 \\
\hline \multicolumn{5}{|c|}{ Manobra da abdução resistida do ombro } \\
\hline MSD & 23 & 52,3 & 21 & 47,7 \\
\hline MSE & 29 & 65,9 & 15 & 34,1 \\
\hline \multicolumn{5}{|c|}{ Manobra de conflito subacromial } \\
\hline MSD & 25 & 56,8 & 19 & 43,2 \\
\hline MSE & 24 & 54,5 & 20 & 45,5 \\
\hline \multicolumn{5}{|l|}{ Ecografia } \\
\hline MSD & 28 & 63,6 & 16 & 36,4 \\
\hline MSE & 27 & 61,4 & 17 & 38,6 \\
\hline Bilateral & - & - & 13 & 29,5 \\
\hline
\end{tabular}

TMR: tendinite do manguito rotador; MSD: membro superior direito; MSE: membro superior esquerdo; Freq.: frequência.

Tabela 4. Resultados da avaliação ecográfica, estratificados por idade.

\begin{tabular}{|c|c|c|c|c|c|c|c|}
\hline \multirow{2}{*}{ Idade } & & \multicolumn{2}{|c|}{ Ecografia MSD } & \multirow{2}{*}{ Total } & \multicolumn{2}{|c|}{ Ecografia MSE } & \multirow{2}{*}{ Total } \\
\hline & & Negativa & Positiva & & Negativa & Positiva & \\
\hline \multirow{4}{*}{$\leq 35$} & Frequências & 12 & 4 & 16 & 12 & 4 & 16 \\
\hline & \% Idade & 75,0 & 25,0 & 100,0 & 75,0 & 25,0 & 100,0 \\
\hline & \% TMR Ecografia & 42,9 & 25,0 & 36,4 & 44,4 & 23,5 & 36,4 \\
\hline & $\%$ do total & 27,3 & 9,1 & 36,4 & 27,3 & 9,1 & 36,4 \\
\hline \multirow{4}{*}{$36-40$} & Frequências & 12 & 4 & 16 & 12 & 4 & 16 \\
\hline & \% Idade & 75,0 & 25,0 & 100,0 & 75,0 & 25,0 & 100,0 \\
\hline & \% TMR Ecografia & 42,9 & 25,0 & 36,4 & 44,4 & 23,5 & 36,4 \\
\hline & $\%$ do total & 27,3 & 9,1 & 36,4 & 27,3 & 9,1 & 36,4 \\
\hline \multirow{4}{*}{$>40$} & Frequências & 4 & 8 & 12 & 3 & 9 & 12 \\
\hline & \% Idade & 33,3 & 66,7 & 100,0 & 25,0 & 75,0 & 100,0 \\
\hline & \% TMR Ecografia & 14,3 & 50,0 & 27,3 & 11,1 & 52,9 & 27,3 \\
\hline & $\%$ do total & 9,1 & 18,2 & 27,3 & 6,8 & 20,5 & 27,3 \\
\hline \multirow{4}{*}{ Total } & Frequências & 28 & 16 & 44 & 27 & 17 & 44 \\
\hline & \% Idade & 63,6 & 36,4 & 100,0 & 61,4 & 38,6 & 100,0 \\
\hline & \% TMR Ecografia & 100,0 & 100,0 & 100,0 & 100,0 & 100,0 & 100,0 \\
\hline & $\%$ do total & 63,6 & 36,4 & 100,0 & 61,4 & 38,6 & 100,0 \\
\hline
\end{tabular}

TMR: tendinite do manguito rotador; MSD: membro superior direito; MSE: membro superior esquerdo. 
autorreferidas e o exame objetivo tanto no membro superior direito (MSD) quanto no membro superior esquerdo (MSE). Dos participantes com queixas autorreferidas ao ombro, apenas 46,2 e 50\% apresentaram, respetivamente, todas as manobras positivas ao exame objetivo, à direita e à esquerda.

Analisou-se, também, a relação existente entre as queixas autorreferidas, cada uma das manobras ao exame objetivo e os resultados da ecografia. A manobra da abdução contrarresistência revelou boa concordância com a sintomatologia $\left(\chi_{\text {Wald }}^{2}(1)=7,260, p=0,007\right)$, apresentando também boa sensibilidade ( $81 \%)$ e especificidade (60,9\%) (Tabela 5).

Esses resultados também foram preditivos considerandose o diagnóstico ecográfico como gold standard $\left(\chi^{2}\right.$ wald $(1)=6,854, p=0,009)$, com ROC $=0,714$, sensibilidade de $75 \%$ e especificidade de $67,9 \%$.

Finalmente, quanto à concordância entre as três metodologias (Tabela 6), a relação é maior quando coexistem: ausência de sintomatologia autorreferida, exame objetivo normal e ecografia sem alterações estruturais.

\section{DISCUSSÃO}

Os resultados da prevalência bilateral de sintomas e sinais de TMR encontrados em ambos os ombros podem dever-se ao facto da atividade de trabalho estar organizada de modo a que haja rotatividade entre caixas "pares" e "ímpares", ou seja, promovendo a alternância de posição corporal relativamente à manipulação dos produtos/cargas e equipamentos de trabalho e, assim, a distribuição do esforço realizado por ambos os membros superiores. Diversos estudos demonstram prevalência elevada de TMR bilateral e de casos assintomáticos ${ }^{1,9,34}$, o que este trabalho veio comprovar.

Efetivamente, a prevalência de TMR bilateral foi considerável, atingindo quase $30 \%$ dos operadores estudados, enquanto a prevalência de casos assintomáticos de TMR situou-se em $22,2 \%$ à direita e em $25,0 \%$ à esquerda. A prevalência considerável de casos assintomáticos de TMR consubstancia a importância e a necessidade da implementação de programas específicos de prevenção a DORT em operadores de caixa que contemplem a informação aos trabalhadores sobre a sua etiologia e a evolução natural desse tipo de

Tabela 5. Sintomatologia autorreferida versus exame objetivo.

\begin{tabular}{l|c|c|c|c}
\multirow{2}{*}{ Manobras exame objetivo } & \multicolumn{4}{c}{ Queixas autorreferidas } \\
\cline { 2 - 5 } & \multicolumn{3}{|c}{ MSD } & \multicolumn{3}{c}{ MSE } \\
\cline { 2 - 6 } Manobra de abdução contrarresistência (\%) & Sensibilidadade & Especificidade & 66,7 & 79,3 \\
\hline Manobra do arco doloroso (\%) & 81,0 & 60,9 & 65,0 & 87,5 \\
\hline Manobra de conflito subacromial (\%) & 81,0 & 60,9 & 65,0 & 87,5 \\
\hline
\end{tabular}

MSD: membro superior direito; MSE: membro superior esquerdo.

Tabela 6. Concordância entre as três metodologias.

\begin{tabular}{|c|c|c|c|c|}
\hline \multirow{2}{*}{ Ultrassonografia } & \multicolumn{2}{|c|}{ MSD } & \multicolumn{2}{|c|}{ MSE } \\
\hline & $\mathrm{n}$ & $\%$ & $\mathrm{n}$ & $\%$ \\
\hline \multicolumn{5}{|l|}{ Sinais de TMR } \\
\hline EO anormal e sintomático & 6 & 13,6 & 7 & 15,9 \\
\hline EO anormal e assintomático & 2 & 4,5 & 0 & 0 \\
\hline EO normal e sintomático & 6 & 13,6 & 3 & 6,8 \\
\hline EO normal e assintomático & 2 & 4,5 & 7 & 15,9 \\
\hline \multicolumn{5}{|l|}{ Avaliação normal } \\
\hline EO anormal e sintomático & 6 & 13,6 & 1 & 2,3 \\
\hline EO anormal e assintomático & 1 & 2,3 & 3 & 6,8 \\
\hline EO normal e sintomático & 8 & 18,2 & 5 & 11,4 \\
\hline EO normal e assintomático & 13 & 29,5 & 18 & 40,9 \\
\hline
\end{tabular}

TMR: tendinite do manguito rotador; EO: exame objetivo; MSD: membro superior direito; MSE: membro superior esquerdo. 
patologia, que, pela sintomatologia inicial fruste, são muitas vezes subvalorizadas pelos doentes até estádios em que a recuperação total nem sempre é possível.

Esses dados devem ser tidos em conta pelos médicos do trabalho, designadamente, no que concerne à periodicidade da vigilância de saúde, devendo ponderar-se o encurtamento do intervalo entre exames periódicos em profissões de risco elevado para o desenvolvimento de TMR.

Com base nos resultados obtidos, talvez a positividade de uma de duas manobras (abdução resistida do ombro ou arco doloroso) possa ter melhor definição para objetivos de rastreio em populações de risco. Apesar dos resultados, e tendo em conta o facto de um estudo ${ }^{7}$ demonstrar o valor preditivo dos sintomas e dos achados ao exame objetivo após um ano de evolução, com proporção maior de participantes com sintomas e/ou alterações ao exame físico na primeira observação a desenvolverem patologia, deverá ser realizada uma vigilância de saúde pouco espaçada no tempo a todos os participantes com queixas autorreferidas e/ou alterações ao exame objetivo. Dever-se-á, ainda, ponderar a implementação de estratégias que visem a melhoria, entre outros, da organização do trabalho e, dessa forma, uma melhor gestão do risco dessa lesão musculoesquelética ligada ao trabalho e, talvez, até estratégias de promoção da saúde no local de trabalho ${ }^{35}$.

\section{CONCLUSÕES}

Os resultados sugerem que a prevalência de DORT difere conforme o método considerado para a sua avaliação. Deve sempre ser considerada a subjetividade - quer das queixas autorreferidas, quer das avaliações clínica e ecográfica muito dependentes do operador —, já que a mesma pode influenciar os resultados.

Os resultados sugerem que, apesar de não existir uma única manobra clínica para o diagnóstico da TMR, a manobra de abdução resistida do ombro, muito simples de executar, pode ser preditiva de TMR na avaliação da saúde de operadores de caixa de supermercado.

O médico do trabalho deve, portanto, no âmbito da vigilância periódica de saúde, utilizar a procura ativa de sintomas e sinais como elementos de diagnóstico precoce - e até de prognóstico - de TMR em operadores de caixa de supermercado, em função dos fatores de risco concretos identificados no exercício dessa atividade.

\section{REFERÊNCIAS}

1. Teefey SA. Shoulder Sonography Why We Do It. J Ultrasound Med. 2012;31(9):1325-31.

2. Dinnes J, Loveman E, Mclntyre L, Waugh N. The effectiveness of diagnostic tests for the assessment of shoulder pain due to soft tissue disorders: a systematic review. Health Technol Assess. 2003;7(29)iii, 1-166.

3. Meroni R, Scelsi M, Boria P, Sansone V. Shoulder disorders in female working-age population: a cross sectional study. BMC Musculoskeletal Disorders. 2014;15(1):118.

4. Lewis JS. Rotator cuff tendinopathy: a review. Brit J Sports Med. 2008.

5. Pope D, Silman A, Cherry N, Pritchard C, Macfarlane G. Association of occupational physical demands and psychosocial working environment with disabling shoulder pain. Ann Rheum Dis. 2001;60(9):852-8.

6. Serranheira F, Uva A, Lopes F. Lesões músculo-esqueléticas e trabalho: alguns métodos de avaliação do risco. Cadernos Avulsos n. ${ }^{0}$ 5. Lisboa: Sociedade Portuguesa de Medicina do Trabalho; 2008.

7. Silverstein BA, Bao SS, Fan ZJ, Howard N, Smith C, Spielholz P, et al. Rotator cuff syndrome: personal, work-related psychosocial and physical load factors. J Occup Environ Med. 2008;50(9):1062-76.

8. Baron SL, Habes D. Occupational musculoskeletal disorders among supermarket cashiers. Scand J Work Environ Health. 1992:127-9.
9. Moosmayer S, Smith H-J, Tariq R, Larmo A. Prevalence and characteristics of asymptomatic tears of the rotator cuff. J Bone Joint Surg Br. 2009;91(2):196-200.

10. Barondess J, Cullen M, de Lateur B, Deyo R, Donaldson K, Drury C, et al. Musculoskeletal disorders and the workplace: Low back and upper extremities. Washington, D.C.: National Academy of Sciences; 2001. 512p.

11. Leclerc A, Chastang J, Niedhammer I, Landre M, Roquelaure Y. Incidence of shoulder pain in repetitive work. Occup Environ Med. 2004;61(1):39-44.

12. Uva A, CarnideF, Serranheira F, Lopes MF, Miranda L. Guia de orientação para prevenção das lesões músculo-esqueléticas e relacionadas com o trabalho: programa nacional contra as doenças reumáticas. Lisboa: Direcção-Geral da Saúde, Ministério da Saúde; 2008.

13. Falcão S, Miguel E, Pimentão J, Branco J, Saraiva F. Ombro. Acta Radiologica Portuguesa. 2008;33:27-30.

14. Van der Windt D, Koes BW, de Jong BA, Bouter LM. Shoulder disorders in general practice: incidence, patient characteristics, and management. Ann Rheum Dis. 1995;54(12):959-64.

15. Östör A, Richards C, Prevost A, Speed C, Hazleman B. Diagnosis and relation to general health of shoulder disorders presenting to primary care. Rheumatol. 2005;44(6):800-5. 
16. Hegedus EJ, Goode AP, Cook CE, Michener L, Myer CA, Myer DM, et al. Which physical examination tests provide clinicians with the most value when examining the shoulder? Update of a systematic review with meta-analysis of individual tests. Brit J Sports Med. 2012;46(14)964-78.

17. Lyons AR, Tomlinson J. Clinical diagnosis of tears of the rotator cuff. J Bone Joint Surg Br. 1992;74(3):414-5.

18. Fodor D, Poanta L, Felea I, Rednic S, Bolosiu H. Shoulder impingement syndrome: correlations between clinical tests and ultrasonographic findings. Ortopedia, Traumatologia, Rehabilitacja. 2008;11(2):120-6.

19. Cardoso A, Branco JC, Silva JAP, Cruz M, Costa MM. Regras de ouro em reumatologia. Lisboa: Direcção-Geral da Saúde; 2005.

20. BurbanK KM, Stevenson JH, Czarnecki GR, Dorfman J. Chronic shoulder pain: part I. Evaluation and diagnosis. Am Fam Physician. 2008;77(4):453-60.

21. Papatheodorou A, Ellinas P, Takis F, Tsanis A, Maris I, Batakis N. US of the Shoulder: Rotator Cuff and Non-Rotator Cuff Disorders 1. Radiographics. 2006;26(1):e23.

22. Kerkovský M, Sprláková-Puková A, Uher T, Vojtaník P, Rouchal M. [Role of ultrasonography in the diagnosis of shoulder joint injury]. Acta Chir Orthop Traumatol Cech. 2008;75(3):167-72.

23. Godefroy D, Sarazin L, Rousselin B, Dupont A, Drape J, Chevrot A. Shoulder imaging: what is the best modality? J Radiol.2001;82(3):317-34.

24. Kayser R, Hampf S, Pankow M, Seeber E, Heyde C. [Validity of ultrasound examinations of disorders of the shoulder joint]. Ultraschall Med. 2005;26(4):291-8.

25. Singh JP. Shoulder ultrasound: what you need to know. Indian J Radiol Imaging. 2012;22(4):284-92.

26. Cruz M, Rosa A, Matos A, Pimentão J, Branco J. Aplicação de ondas de choque extra-corpóreas no tratamento da tendinite calcificante do ombro. Acta Reumatol Portuguesa. 2003;28:45-51.

27. Reilly P, Macleod I, Macfarlane R, Windley J, Emery R. Dead men and radiologists don't lie: a review of cadaveric and radiological studies of rotator cuff tear prevalence. Ann R Coll Surg Eng. 2006;88(2):116-21.
28. Petranova T, Vlad V, Porta F, Radunovic G, Micu MC, Nestorova R, et al. Ultrasound of the shoulder. Med Ultrason. 2012;14(2):133-40.

29. Jacobson JA, Lancaster S, Prasad A, van Holsbeeck MT, Craig JG, Kolowich P. Full-Thickness and Partial-Thickness Supraspinatus Tendon Tears: Value of US Signs in Diagnosis 1. Radiol. 2004;230(1):234-42.

30. Beall MH, Spong CY, Ross MG. A randomized controlled trial of prophylactic maneuvers to reduce head to body delivery time in patients at risk for shoulder dystocia. Obstet Gynecol. 2003;102(1):31-5.

31. Barbieri P, Pizzoni T, Scolari L, Lucchini R. [Symptoms and upper limb work-related musculo-skeletal disorders among 173 supermarket cashiers]. Med Lav. 2013;104(3):236-43.

32. Serranheira F, Pereira M, Santos C, Cabrita M. Auto-referência de sintomas de LME numa grande empresa em Portugal. Rev Portug Saúde Pública. 2003;2:37-48.

33. Serranheira F, Cotrim T, Rodrigues V, Nunes C, Sousa-Uva A. Lesões musculoesqueléticas ligadas ao trabalho em enfermeiros portugueses: «ossos do ofício» ou doenças relacionadas com o trabalho? Rev Portug Saúde Pública. 2012;30(2):193-203.

34. Sanábio E, Carneiro T, Couto H. Diagnóstico médico com ultra-som: problemas ergonômicos dos profissionais envolvidos. Rev Bras Med Trab. 2003;1(1):22-30.

35. Sousa-Uva A, Serranheira F. Trabalho e Saúde/Doença: o desafio sistemático da prevenção dos riscos profissionais e o esquecimento reiterado da promoção da saúde. Rev Bras Med Trab. 2013;11(1):43-9.

Endereço para correspondência: Rita Silva Pereira - Avenida Dom João II, Lote 4.69.01, Serviço de Saúde Ocupacional IPL - CEP: 1990-096 - Lisboa, Portugal - E-mail: ritamsilvapereira@gmail.com 